\title{
Phase II clinical trial of adoptive cell therapy for patients with metastatic melanoma with autologous tumor-infiltrating lymphocytes and low-dose interleukin-2
}

\author{
Linh T. Nguyen ${ }^{1}$ (D) Samuel D. Saibil ${ }^{2} \cdot$ Valentin Sotov $^{1} \cdot$ Michael X. Le $^{1} \cdot$ Leila Khoja $^{2} \cdot$ Danny Ghazarian $^{3}$. \\ Luisa Bonilla ${ }^{2} \cdot$ Habeeb Majeed $^{2} \cdot$ David Hogg $^{2} \cdot$ Anthony M. Joshua ${ }^{2,4} \cdot$ Michael Crump $^{2} \cdot$ Norman Franke $^{2}$. \\ Anna Spreafico ${ }^{2}$ - Aaron Hansen ${ }^{2}$ - Ayman Al-Habeeb ${ }^{3}$. Wey Leong ${ }^{5}$. Alexandra Easson ${ }^{5}$ - Michael Reedijk ${ }^{5}$. \\ David P. Goldstein ${ }^{5,6}$. David McCready ${ }^{5} \cdot$ Kazuhiro Yasufuku $^{5}$. Thomas Waddell ${ }^{5} \cdot$ Marcelo Cypel $^{5} \cdot$ Andrew Pierre $^{5}$. \\ Bianzheng Zhang ${ }^{2}$. Sarah Boross-Harmer ${ }^{1}$. Jane Cipollone ${ }^{1} \cdot$ Megan Nelles $^{1} \cdot$ Elizabeth Scheid $^{1} \cdot$ Michael Fyrsta $^{1}$. \\ Charlotte S. Lo ${ }^{1}$. Jessica Nie ${ }^{1}$. Jennifer Y. Yam ${ }^{1}$ - Pei Hua Yen ${ }^{1}$. Diana Gray ${ }^{1}$. Vinicius Motta ${ }^{1}$. Alisha R. Elford ${ }^{1}$. \\ Stephanie DeLuca ${ }^{7} \cdot$ Lisa Wang $^{8} \cdot$ Stephanie Effendi $^{8} \cdot$ Ragitha Ellenchery $^{8} \cdot$ Naoto Hirano $^{1,9} \cdot$ Pamela S. Ohashi $^{1,9}$. \\ Marcus O. Butler ${ }^{1,2,9,10}$
}

Received: 7 June 2018 / Accepted: 17 January 2019 / Published online: 11 February 2019

(c) The Author(s) 2019

\begin{abstract}
Adoptive cell therapy using autologous tumor-infiltrating lymphocytes (TIL) has shown significant clinical benefit, but is limited by toxicities due to a requirement for post-infusion interleukin-2 (IL-2), for which high dose is standard. To assess a modified TIL protocol using lower dose IL-2, we performed a single institution phase II protocol in unresectable, metastatic melanoma. The primary endpoint was response rate. Secondary endpoints were safety and assessment of immune correlates following TIL infusion. Twelve metastatic melanoma patients were treated with non-myeloablative lymphodepleting chemotherapy, TIL, and low-dose subcutaneous IL-2 (125,000 IU/kg/day, maximum 9-10 doses over 2 weeks). All but one patient had previously progressed after treatment with immune checkpoint inhibitors. No unexpected adverse events were observed, and patients received an average of 6.8 doses of IL-2. By RECIST v1.1, two patients experienced a partial response, one patient had an unconfirmed partial response, and six had stable disease. Biomarker assessment confirmed an increase in IL-15 levels following lymphodepleting chemotherapy as expected and a lack of peripheral regulatory T-cell expansion following protocol treatment. Interrogation of the TIL infusion product and monitoring of the peripheral blood following infusion suggested engraftment of TIL. In one responding patient, a population of T cells expressing a T-cell receptor V $\beta$ chain that was dominant in the infusion product was present at a high percentage in peripheral blood more than 2 years after TIL infusion. This study shows that this protocol of low-dose IL-2 following adoptive cell transfer of TIL is feasible and clinically active. (ClinicalTrials.gov identifier NCT01883323.)
\end{abstract}

Keywords Tumor-infiltrating lymphocytes · Adoptive cell therapy $\cdot$ Metastatic melanoma $\cdot$ Interleukin- $2 \cdot$ Clinical trial

\section{Abbreviations}

AJCC American Joint Committee on Cancer

CR Complete response

Electronic supplementary material The online version of this article (https://doi.org/10.1007/s00262-019-02307-x) contains supplementary material, which is available to authorized users.

Marcus O. Butler

Marcus.Butler@uhn.ca

Extended author information available on the last page of the article
CTCAE Common terminology criteria for adverse events

ECOG Eastern Cooperative Oncology Group

G-CSF Granulocyte colony-stimulating factor

irRC Immune-Related Response Criteria

MART-1 Melanoma antigen recognized by T cells 1

PD Progressive disease

PR Partial response

$\mathrm{PRu} \quad$ Unconfirmed partial response

REP Rapid expansion protocol

SD Stable disease 


\section{Introduction}

Adoptive cell therapy (ACT) using autologous tumor-infiltrating lymphocytes (TIL) has demonstrated tremendous potential for treatment of advanced tumors, particularly melanoma (Reviewed in [1]). Current ACT protocols incorporate the use of preparative non-myeloablative lymphodepleting chemotherapy regimens before the infusion of the ex vivo expanded autologous TIL. Subsequent to the TIL infusion, patients are treated with high-dose interleukin-2 (IL-2). Using this approach, objective response rates ranging from 38 to $50 \%$ have been reported in patients with metastatic melanoma, with durable, complete tumor regression observed in up to $20 \%$ of patients [2-6]. These data indicate that TIL therapy offers the potential for durable clinical benefit, even in patients with few treatment options.

The tradeoff for these encouraging results is that current treatment protocols of TIL therapy result in a high rate of grade 3 or 4 toxicities. The preparative chemotherapy has been demonstrated to increase the efficacy of therapy, in part by increasing the availability of homeostatic cytokines, such as IL-15, for the infused TIL as well as by depleting T-regulatory cells (Tregs) [7-10]. Unfortunately, the preparative chemotherapy also commonly results in anemia and thrombocytopenia requiring transfusions and sustained neutropenia requiring growth factor support [7]. The IL-2 given post-TIL infusion, to support survival and expansion of the transferred TIL, has typically been administered as highdose, intravenous IL-2 at a dose of 720,000 IU/kg every $8 \mathrm{~h}$ as tolerated for a maximum of 5 days [2-5]. High-dose IL-2 is also associated with significant toxicity including neurological symptoms and a systemic capillary leak syndrome that can result in significant hypotension, renal failure, and pulmonary edema requiring intensive care unit (ICU) care [11]. Attempts have been made to alter the current TIL protocols to limit this toxicity via modifications of the IL-2 dosing regimen post-TIL infusion [12-14]. A pilot study of subcutaneously administered IL-2 at a dose of 2 MIU/day for 14 days demonstrated durable clinical responses in two of the six patients [13]. In addition, a larger trial of 25 patients demonstrated an objective response rate of $42 \%$ utilizing a "decrescendo" regimen of continuous, intravenous IL-2 at a dose of $18 \mathrm{MIU} / \mathrm{m}^{2}$ over 6,12 and then $24 \mathrm{~h}$ followed by 4.5 $\mathrm{MIU} / \mathrm{m}^{2}$ over $24 \mathrm{~h}$ for 3 days [14]. These data indicated that high-dose IL-2 is not an absolute requirement to derive clinical benefit from TIL therapy and that further investigations into modified IL-2 dosing regimens are warranted. Here, we report the results of a phase II study of 12 patients with metastatic melanoma treated with a modified ACT protocol utilizing autologous TIL with preconditioning chemotherapy followed by the administration of subcutaneous IL-2 administered at a low dose of $125,000 \mathrm{IU} / \mathrm{kg} /$ day over 12 days.

\section{Materials and methods}

\section{Patients}

All patients underwent informed consent to participate in this study. Patients 18 years or older with unresectable stage III or stage IV melanoma, per the 7 th edition of the AJCC staging [15], were eligible for enrollment. Other requirements were an Eastern Cooperative Oncology Group (ECOG) performance status of 0 or 1 and a life expectancy of greater than 3 months from the date of consent to TIL treatment. Patients with brain metastases were eligible provided that they had three or fewer asymptomatic lesions each measuring less than or equal to $1 \mathrm{~cm}$. Alternatively, patients with brain metastases not meeting these criteria were eligible if they had definitive treatment with surgery and/or radiation at least 30 days prior to the first dose of lymphodepleting chemotherapy. Key exclusion criteria were active chronic infections, continuing requirement for systemic corticosteroid treatment as well as significant medical comorbidities including active cardiac illness or pulmonary dysfunction.

\section{Study design}

The primary endpoint of this study was clinical efficacy as defined by response according to the Response Evaluation Criteria in Solid Tumors (RECIST) guideline (version 1.1) [16]. Response was also assessed using the Immune-related Response Criteria (irRC) [17]. Secondary endpoints were to evaluate the safety of this low-dose IL-2 treatment protocol and to evaluate the immune status of patients following treatment. Toxicity was assessed using the Common Terminology Criteria for Adverse Events (CTCAE) version 4.0.

All patients received a non-myeloablative, lymphodepleting chemotherapy regimen consisting of cyclophosphamide $(60 \mathrm{mg} / \mathrm{kg} / \mathrm{day})$ for 2 days (days -5 and -4 ) as well as fludarabine $\left(25 \mathrm{mg} / \mathrm{m}^{2} /\right.$ day) for 5 days (days -5 to -1 ) as an inpatient before TIL infusion. At least 1 week prior to the initiation of this preparative chemotherapy, all patients also underwent mobilization with filgrastim and leukapheresis to cryopreserve hematopoietic stem cells. TIL were infused on day 0 of the protocol, and on the same day, subcutaneous IL-2 was initiated at a dose of 125,000 IU/kg/day. IL-2 was administered daily as an inpatient over a 12-day period with a 2-day break after the first 4-5 doses of IL-2 (maximum 9-10 doses). The regimen was based on the low-dose regimen described by Yang et al. [18], and modified with the aim of further reducing toxicities. All patients were treated with daily filgrastim injections as well as ciprofloxacin and cloxacillin, while absolute neutrophil count (ANC) was less than $1.0 \times 10^{9} / \mathrm{L}$. Prophylaxis with acyclovir and fluconazole was initiated at the end of chemotherapy. Prophylactic 
trimethoprim and sulfamethoxazole were initiated upon hospitalization and continued until absolute CD4+ count was above $0.2 \times 10^{9} / \mathrm{L}$ and for at least 6 months after chemotherapy. Platelet and red blood cell transfusions were given as needed to maintain platelets greater than $10 \times 10^{9} / \mathrm{L}$ and hemoglobin greater than $80 \mathrm{~g} / \mathrm{L}$.

\section{TIL culturing}

The TIL manufacturing procedure was adapted from Dudley et al. [19]. Melanoma tissue was processed by cutting into $\sim 1 \mathrm{~mm}^{3}$ fragments. Tissue fragments were either plated directly into 24 -well plates or enzymatically dissociated in medium comprised of Iscove's Modified Dulbecco's Medium (IMDM) (Lonza) containing Collagenase (Sigma) and Pulmozyme (Roche) and then plated in 24-well plates at a concentration of $1.0 \times 10^{6}$ cells/well. Cells were cultured in complete medium comprised of: IMDM (Lonza), 10\% healthy donor plasma (prepared in-house, as described in Nguyen et al. [20]), HEPES (Lonza), penicillin/streptomycin (Lonza) (omitted for patients with penicillin allergy), gentamycin (Lonza), 2-mercaptoethanol (Invitrogen), L-glutamine (Lonza), and 6,000 IU/mL IL-2 (Proleukin, Novartis). Cells were maintained at a concentration of approximately $1.0 \times 10^{6}$ cells $/ \mathrm{mL}$ and expanded for a maximum of 28 days prior to cryopreservation and QC testing. In general, four independent, bulk TIL cultures were established from each patient specimen.

\section{Rapid expansion protocol (REP)}

TIL from two independent bulk TIL cultures were thawed, rested, and seeded in parallel in Rapid Expansion Protocols (REPs) with $30 \mathrm{ng} / \mathrm{mL}$ OKT3 (GMP Grade) (Miltenyi Biotec), irradiated (50 Gy) allogeneic PBMC feeder cells (1:100 TIL:PBMC), and 600 IU/mL of IL-2 in " $50 / 50$ " media containing 50\% complete medium (as described above) prepared using human serum $\mathrm{AB}+$ (Gemini Bio Products) and 50\% AIM V media (Invitrogen). Fungizone (Lonza) was added on day 5 onwards. Note that a lower concentration of IL-2 was used in the REP compared to the protocol developed by Dr. Steven Rosenberg (Surgery Branch, National Cancer Institute). The REP was performed in G-Rex 100 flasks (Wilson Wolf) for all patients except for the first patient treated. For Patient 1, the REP was initiated in T175 flasks and then transferred to $3 \mathrm{~L}$ culture bags (Fenwal) on day 7. TIL were harvested on day 14 of the REP for all patients except Patient 5 , whose TIL were harvested on day 15 due to a change in the clinical schedule. The final product was prepared in infusion media comprised of $2.5 \%$ human serum albumin, $300 \mathrm{IU} / \mathrm{mL}$ IL-2 in $0.9 \%$ saline (Baxter) and transferred to a transfer pack (Fenwal) for infusion.
For TIL manufacturing and $\mathrm{QC}$, percent $\mathrm{CD}^{+}$was assessed by flow cytometry (clone UCHT1). Two independent bulk cultures of TIL were selected for the REP based on features such as enrichment for $\mathrm{CD} 8^{+}$cells and in vitro tumor reactivity; however, TIL cultures selected for the REP were not required to satisfy any criteria related to $\% \mathrm{CD}^{+}$ cells or tumor reactivity.

\section{Tumor reactivity assays}

The tumor reactivity of pre-REP TIL was assayed in vitro when possible. IFN- $\gamma$ production was assessed by co-culturing TIL with target cells overnight and assaying the supernatant by ELISA (eBioscience/Thermo). Positive IFN- $\gamma$ activity was defined as secretion of $>200 \mathrm{pg} / \mathrm{mL}$ IFN- $\gamma$ above baseline (TIL alone) with at least a $50 \%$ decrease in IFN- $\gamma$ secretion in the presence of anti-HLA Class I antibody (clone W6/32). Target cells were cryopreserved, enzyme-dissociated, autologous tumor in all cases except for Patient 9, where MART1/Melan-A-peptide-loaded HLAA*02:01 + lymphoblastoid (T2) or HLA-A-matched or mismatched melanoma cell lines (526mel, 888mel, respectively; kind gifts from Dr. S. Rosenberg) were used as surrogate targets. Cytotoxicity (CTL) assays were performed by standard chromium $\left({ }^{51} \mathrm{Cr}\right)$ release assay. TIL were considered to be tumor reactive if all the following criteria were satisfied: (1)>10\% specific lysis at either one of a $60: 1$ or $20: 1$ effector:target ratio; (2) an overall decrease in specific lysis with each decrease in effector:target ratio; and (3) a $>25 \%$ reduction in specific lysis in the presence of anti-HLA Class I antibody (clone W6/32) compared to specific lysis in the absence of W6/32 at an effector:target ratio of either 60:1 or $20: 1$.

\section{Microbead immunoassay}

Concentrations of cytokines were measured in serum using the Bio-Plex Pro Human Cytokine 27-plex Assay (Bio-Rad) per manufacturer's instructions. Serum obtained from 10 healthy donors was also tested to establish a normal range for each cytokine. Samples were run and analyzed using the Bio-Plex MAGPIX multiplex reader.

\section{Flow cytometry analysis}

Phenotypic analysis was performed on freshly isolated PBMC using the following monoclonal antibodies. From Fisher Scientific: PD-1 (J105), CD4 (RPA-T4), FoxP3 (236A/E7), CD127 (eBioRDR5), CD25 (4E3); from BioLegend: CD8 (SK1), CD3 (UCHT1), CD14 (M5E2), and CD19 (H1B19). The TCR V $\beta$ repertoire was analyzed using the IOTest Beta Mark Kit (Beckman-Coulter). Staining for MART-1/Melan-A [26-35 (27L); ELAGIGILTV] specific T 
cells was performed using APC-bound pentamers (ProImmune). Intracellular staining (FoxP3) was performed after applying fixation/permeabilization buffer (Fisher Scientific). All samples were run on a Canto II flow cytometer and analyzed using FlowJo Ver. 9.5.8. Dominant TCR V $\beta$ populations were identified based on any $\mathrm{V} \beta$ chain whose frequency was considered to be a statistical outlier in the repertoire of the $24 \mathrm{~V} \beta$ chains that were analyzed. An outlier test was used to define a $\mathrm{V} \beta$ as dominant if its frequency was at least three interquartile distances away from the third quartile of all the $\mathrm{V} \beta$ chains analyzed.

\section{Statistics}

A two-stage Simon design for a phase II trial was followed: A response rate of $5 \%$ or lower was considered too low to be of interest $(\mathrm{p} 0=0.05)$, while if the treatment had a response rate of $30 \%$ or more, it was considered to be effective $(\mathrm{pA}=0.3)$. A total of 12 patients were required to be accrued to this study with an overall probability to reject an effective treatment of 0.198 and an overall probability to accept an ineffective treatment of 0.084 . Accrual was conducted in stages. During the first stage, five patients were accrued. If there were no responders, then the study would have stopped with the conclusion that treatment was ineffective. If there was at least one responder, then the study would continue to the second stage and accrue seven more patients to a total of 12 . If by the end of the study, there were one or less responders in the total of 12 patients, the treatment would be considered ineffective. If there were two or more, then the treatment would be considered effective.

\section{Results}

\section{Patient and TIL characteristics}

Between October 2013 and December 2017, 12 patients with metastatic melanoma were treated on protocol. Within this cohort, one patient had a melanoma of ocular origin, two had mucosal melanomas, and the remaining had cutaneous melanoma. All of the patients except one had M1c disease, as per the American Joint Committee on Cancer (AJCC) 7th edition classification, with the remaining patient having M1b disease. Four had previously-treated brain metastases before enrollment in the trial. All patients had been heavily pretreated for their metastatic disease, with the exception of one patient who did not receive prior therapy. Ten of the 12 patients had received prior treatment with ipilimumab and nine had received prior programmed death-1 (PD-1) blockade with either nivolumab or pembrolizumab. Two patients also had received combination immunotherapy with combined ipilimumab and nivolumab. In addition, eight patients

Table 1 Patient characteristics

\begin{tabular}{|c|c|c|c|c|c|c|c|}
\hline Patients & Sex & Age & Histology & BRAF status & M stage ${ }^{a}$ & Disease sites & Previous treatment \\
\hline 1 & $\mathrm{M}$ & 43 & Cutaneous & WT & M1b & LN, SC, lung & None \\
\hline 2 & $\mathrm{M}$ & 64 & Cutaneous & WT & M1c & LN, lung, liver, adrenal & Ipi/Nivo, Carbo-tax \\
\hline 3 & $\mathrm{~F}$ & 35 & Cutaneous & WT & M1c & Lung, Sp, peritoneum, bowel & Carbo-tax, Ipi \\
\hline 4 & $\mathrm{M}$ & 48 & Cutaneous & V600E & M1c & $\begin{array}{l}\text { Brain, LN, lung, Sp, kidney, gallbladder, } \\
\text { psoas }\end{array}$ & Dabrafenib/Trametinib, Ipi, Pembro \\
\hline 5 & $\mathrm{~F}$ & 40 & Mucosal & WT & M1c & SC, lung, liver, kidney, retroperitoneum & Carbo-tax, Ipi, DTIC \\
\hline 6 & $\mathrm{~F}$ & 49 & Mucosal & WT & M1c & LN, lung, pleura, uterus, bone & Ipi, Pembro, Carbo-tax \\
\hline 7 & M & 49 & Cutaneous & WT & M1c & $\begin{array}{l}\text { Brain, LN, SC, lung, pleura, chest wall, } \\
\text { liver, Sp, small bowel }\end{array}$ & Ipi, Pembro \\
\hline 8 & M & 35 & Cutaneous & WT & M1c & $\begin{array}{l}\text { LN, SC, lung, Sp, kidney, bone, ureter, } \\
\text { pancreas }\end{array}$ & DTIC, Ipi, Pembro, Carbo-tax \\
\hline 9 & $\mathrm{~F}$ & 34 & Cutaneous & WT & M1c & $\begin{array}{l}\text { LN, SC, lung, peritoneum, liver, kidney, } \\
\text { breast }\end{array}$ & DTIC, Ipi, Pembro, IL-2 (injections) \\
\hline 10 & M & 61 & Cutaneous & WT & M1c & $\begin{array}{l}\text { Brain, LN, lung, kidney, pleura, per- } \\
\text { inephric space }\end{array}$ & Ipi/Nivo, Pembro \\
\hline 11 & M & 42 & Uveal & WT & M1c & $\begin{array}{l}\text { LN, SC, lung, peritoneum, liver, kidney, } \\
\text { pleura }\end{array}$ & DTIC/Selumetinib, Ipi/Nivo, Pembro \\
\hline 12 & M & 61 & Cutaneous & WT & M1c & $\begin{array}{l}\text { Brain, LN, SC, lung, peritoneum, liver, } \\
\text { pericardium, adrenal gland }\end{array}$ & Nivo, anti-PD-1/anti-GITR, Carbo-tax \\
\hline
\end{tabular}

WT wild type, $L N$ lymph nodes, SC subcutaneous, Sp spleen, Ipi ipilimumab, Nivo nivolumab, Carbo-tax carboplatin + paclitaxel, Pembro pembrolizumab, DTIC dacarbazine

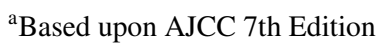


had been treated with chemotherapy. The baseline characteristics of the patients are summarized in Table 1.

The method for in vitro TIL expansion was adapted from Dr. Steven Rosenberg (NCI) [19]. Of note, the concentration of IL-2 that we used during the second stage of expansion (Rapid Expansion Protocol; REP) (600 IU/mL) was lower than that used by the Rosenberg group and commonly used in other TIL protocols $(3000 \mathrm{IU} / \mathrm{mL})$. The characteristics of the TIL cultures and the infusion products are summarized in Table 2. TIL were harvested from sites other than lymph nodes in 8 of 12 patients. The average fold expansion during the REP was 2619 fold (range 1159-4700). This expansion allowed an average of $1.12 \times 10^{11}$ cells to be infused for treatment (range $5.5 \times 10^{10}-1.6 \times 10^{11}$ ). The percentage of $\mathrm{CD} 8^{+}$versus $\mathrm{CD} 4^{+} \mathrm{T}$ cells in each infusion product was determined. Eight of the 12 infusion products (Patients 1, 4, 6-8, and 10-12) had a CD8 ${ }^{+}$dominant phenotype with greater than $60 \% \mathrm{CD}^{+} \mathrm{T}$ cells. Patient 5 received a CD $4^{+}$dominant TIL product, with greater than $60 \%$ being $\mathrm{CD} 4^{+}$lymphocytes. The remaining three patients (Patients 2,3 , and 9) comprised a third group, receiving more balanced infusion products in which neither T-cell population exceeded $60 \%$.

\section{Treatment-related toxicity}

The incidence and severity of each treatment-related adverse event observed during the trial are summarized in Table 3. There were no grade 5 adverse events related to study therapy. All of the patients experienced grade 3-4 hematological toxicities as expected with lymphodepleting chemotherapy. In ten patients, these toxicities were reversible with blood and platelet transfusions and G-CSF growth factor support. One patient, however, experienced refractory pancytopenia with marrow aplasia found on bone marrow biopsy. Infusion of the patient's preserved hematopoietic stem cells restored hematopoiesis and the patient's cell counts subsequently recovered. A second patient also received stem cells on day 13 for delayed recovery of cell counts in the setting of extensive melanoma bone metastasis and marrow involvement. This patient's cell counts recovered with no infectious or bleeding complications.

Febrile neutropenia was also a commonly observed toxicity in these patients after lymphodepletion. In six patients, no infectious source for the fever was ever identified. Conversely, the patient with aplastic bone marrow also developed multiple infectious complications including a positive nasopharyngeal swab for respiratory syncytial virus for which oral ribavirin therapy was initiated and Streptococcus viridans and Klebsiella oxytoca bacteremia that was treated with antibiotics.

Low-dose IL-2 was reasonably well tolerated with patients receiving an average of 6.8 doses (range from 2 to 9 doses). The majority of the toxicities directly attributable to IL-2 were grade 1 or 2 and were those relating to vascular leak syndrome, including peripheral edema, pulmonary edema, hypotension and increased creatinine or decreased urine output, as well as fever, fatigue and neurological symptoms. In most patients, these symptoms could be managed with supportive measures and by delaying or omitting subsequent IL-2 injections. Eight of the 12 patients were able to receive at least seven doses of the targeted nine doses of IL-2 and five patients received all nine doses. Three patients only received four injections. One of these patients developed a grade 3 pruritic rash that was determined to be related to trimethoprim and sulfamethoxazole, and as a precaution, further IL-2 injections were held. Of the other two patients who only received four doses of IL-2, one developed grade
Table 2 Pre-REP TIL and TIL infusion product characteristics

\begin{tabular}{lllllrr}
\hline Patients & $\begin{array}{l}\text { Tumor specimen } \\
\text { from LN? }\end{array}$ & $\begin{array}{l}\text { Days in pre-REP cul- } \\
\text { ture (per TIL culture) }\end{array}$ & $\begin{array}{l}\text { REP fold- } \\
\text { expansion }\end{array}$ & $\begin{array}{l}\text { Number of } \\
\text { cells infused }\end{array}$ & \multicolumn{2}{l}{\begin{tabular}{l} 
Infusion product \\
\cline { 5 - 6 } \%CD8
\end{tabular}} \\
\hline 1 & N & 21,22 & 1159 & $5.50 \times 10^{10}$ & 82.0 & 5.4 \\
2 & $\mathrm{Y}$ & 14,14 & 1700 & $8.60 \times 10^{10}$ & 44.8 & 54.1 \\
3 & $\mathrm{Y}$ & 16,17 & 2040 & $1.00 \times 10^{11}$ & 48.2 & 39.4 \\
4 & $\mathrm{Y}$ & 18,18 & 2640 & $1.32 \times 10^{11}$ & 85.3 & 10.5 \\
5 & $\mathrm{~N}$ & 20,20 & 1524 & $8.00 \times 10^{10}$ & 30.2 & 69.3 \\
6 & $\mathrm{Y}$ & 14,17 & 2750 & $1.06 \times 10^{11}$ & 80.2 & 15.5 \\
7 & $\mathrm{~N}$ & 14,14 & 2917 & $1.46 \times 10^{11}$ & 93.0 & 6.2 \\
8 & $\mathrm{~N}$ & 17,17 & 4700 & $1.60 \times 10^{11}$ & 62.3 & 35.2 \\
9 & $\mathrm{~N}$ & 16,16 & 2560 & $8.00 \times 10^{10}$ & 47.9 & 39.5 \\
10 & $\mathrm{~N}$ & 16,16 & 4452 & $1.56 \times 10^{11}$ & 96.1 & 3.7 \\
11 & $\mathrm{~N}$ & 22,26 & 2017 & $9.84 \times 10^{10}$ & 81.2 & 18.2 \\
12 & $\mathrm{~N}$ & 18,18 & 2972 & $1.46 \times 10^{11}$ & 73.3 & 21.4 \\
\hline
\end{tabular}

$L N$ lymph node, $T I L$ tumor-infiltrating lymphocyte, $R E P$ Rapid Expansion Protocol 
Table 3 Adverse events related to treatment

\begin{tabular}{|c|c|c|c|c|}
\hline \multirow[t]{2}{*}{ Toxicities } & \multicolumn{4}{|c|}{ Number (maximum grade) } \\
\hline & Grade 1 & Grade 2 & Grade 3 & Grade 4 \\
\hline \multicolumn{5}{|l|}{ Hematologic } \\
\hline Neutropenia & & & 5 & 7 \\
\hline Thrombocytopenia & & & 6 & 5 \\
\hline Leukopenia & & & 11 & \\
\hline Lymphopenia & & 1 & 2 & 4 \\
\hline Anemia & 1 & & 10 & \\
\hline Bone marrow aplasia/delayed engraftment & & & 2 & \\
\hline Febrile neutropenia & & & 7 & \\
\hline \multicolumn{5}{|l|}{ Gastrointestinal } \\
\hline LFT increase & 7 & 1 & & \\
\hline Bilirubin & 1 & 2 & & \\
\hline Nausea/vomiting & 6 & 9 & & \\
\hline Diarrhea & 4 & 6 & & \\
\hline Pain & 4 & 3 & & \\
\hline Mucositis & 1 & 1 & & \\
\hline Constipation & 1 & 1 & & \\
\hline Anorexia & 3 & 2 & & \\
\hline Blood in stool and melena & & 2 & & \\
\hline \multicolumn{5}{|l|}{ Renal } \\
\hline Electrolyte imbalance & 7 & 5 & 3 & \\
\hline AKI creatinine & 6 & & & \\
\hline Hematuria & 4 & 2 & & \\
\hline Urine output decreased & & & 3 & \\
\hline Urinary retention & & & 2 & \\
\hline Proteinuria & 1 & & & \\
\hline \multicolumn{5}{|l|}{ Neurologic } \\
\hline Fatigue & 3 & 4 & 1 & \\
\hline Headache & 2 & 3 & & \\
\hline Hallucinations & 1 & & & \\
\hline Insomnia & 2 & & & \\
\hline Paresthesia & 1 & & & \\
\hline Drowsiness and mental status change & 2 & & & \\
\hline \multicolumn{5}{|l|}{ Musculoskeletal } \\
\hline Pain & 3 & 1 & & \\
\hline CPK increased & 1 & & & \\
\hline Arthralgia & & 1 & & \\
\hline \multicolumn{5}{|l|}{ Vascular leak syndrome } \\
\hline Edema, weight gain & 10 & 3 & 1 & \\
\hline Pulmonary edema & & 2 & & \\
\hline Hypotension & 1 & 1 & & \\
\hline Dyspnea respiratory distress & 3 & 4 & 1 & \\
\hline Hypoxia & & 2 & 3 & \\
\hline Capillary leak syndrome & & 3 & & \\
\hline Pleural effusion & 1 & & & \\
\hline Pericardial effusion & & 1 & & \\
\hline \multicolumn{5}{|l|}{ Dermatologic } \\
\hline Rash & 4 & 2 & 1 & \\
\hline Pruritus & 1 & & & \\
\hline Alopecia & 2 & & & \\
\hline
\end{tabular}


Table 3 (continued)

\begin{tabular}{|c|c|c|c|c|}
\hline \multirow[t]{2}{*}{ Toxicities } & \multicolumn{4}{|c|}{ Number (maximum grade) } \\
\hline & Grade 1 & Grade 2 & Grade 3 & Grade 4 \\
\hline Skin hypopigmentation (Vitiligo) & 2 & & & \\
\hline \multicolumn{5}{|l|}{ Infections } \\
\hline Fever & 4 & 5 & & \\
\hline EBV infection & 1 & & & \\
\hline RSV viral infection & 1 & & & \\
\hline Pleural infection & & 1 & & \\
\hline Upper respiratory infection & & 1 & & \\
\hline
\end{tabular}

$L F T$ liver function test, $A K I$ acute kidney injury, $C P K$ creatine phosphokinase test, $E B V$ Epstein-Barr virus, $R S V$ respiratory syncytial virus

3 edema, and the other developed grade 2 edema and grade 1 renal failure that resolved with the discontinuation of IL-2. Patient 9, who had progressive melanoma metastases, received only two doses of IL-2 due to IL-2-induced fluid retention. Nine days following the last dose of IL-2, this patient went on to develop pulmonary infiltrates, hypoxia, and respiratory distress requiring temporary mechanical ventilation. This episode was attributed to an immune reconstitution syndrome rather than the previous IL-2. Overall, most patients were able to receive six or more doses of low-dose IL-2 with acceptable toxicities that could be managed in the non-ICU setting.

The other grade 3 toxicities observed were either related to the chemotherapy, such as electrolyte imbalances, or to the infusion of TIL. Two patients experienced hypoxic episodes immediately following infusion of TIL. In both cases, these episodes were transient and resolved with supportive treatment, but did result in a delay in starting IL-2.

\section{Clinical efficacy}

When clinical response was assessed using RECIST v1.1 criteria, two patients (Patients 1 and 8) achieved a partial response (PR) and one patient (Patient 7) had an unconfirmed partial response (PRu). The rest of the patients had either stable disease (SD) lasting less than 6 months or progressive disease (PD) at the time of the first assessment (6 SD, 3 PD). Per RECIST v1.1 criteria, the estimated median progression free survival was 5.1 months (95\%CI: 1.2-6.4 months). The estimated median overall survival was estimated to be 6.2 months (1.5 months-not reached), with five deaths being observed on trial (Supplementary Fig. 1). Applying the irRC criteria increased the number of confirmed PRs to three, as the patient with a PRu according to RECIST had continued decrease in the size of total lesion burden despite the presence of new liver lesions. This patient unfortunately had rapid progression of these liver lesions as well as disease elsewhere, including cutaneous lesions, shortly after having his PR confirmed. In the rest of the patients, we did not observe any evidence of late responses or pseudoprogression. A caveat, however, was that few patients had the requisite repeat imaging studies to confirm progression by irRC, because most patients had significant clinical progression that necessitated starting subsequent therapies before these studies could be obtained.

Despite only achieving clinical responses in 3 of the 12 patients, there was evidence of anti-tumor activity of the TIL in two additional patients. As depicted in the waterfall plot in Fig. 1, Patients 4 and 6 had tumor regression of greater than $10 \%$; exhibiting $25 \%$, and $14 \%$ reduction of target lesions, respectively. In all of these patients, the observed peak regression of target lesions was documented on the first radiographic assessment after treatment, with subsequent progression.

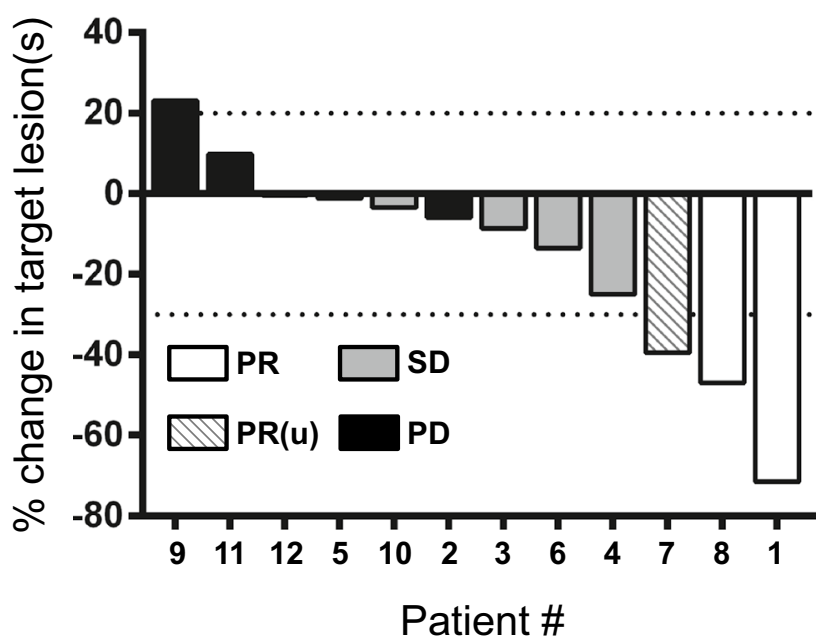

Fig. 1 Waterfall plot. The best percentage change in the target lesion(s) is shown for each patient. The dotted lines show the threshold for progressive disease versus stable disease of target lesions $(+20 \%)$ and partial response versus stable disease of target lesions (- 30\%). The best response by RECIST v1.1 is shown for each patient. $P R$ partial response, $P R(u)$ unconfirmed partial response, $S D$ stable disease, $P D$ progressive disease 


\section{Immune monitoring and TIL characteristics}

Multiple immunological parameters were monitored throughout the protocol. The serum levels of the homeostatic cytokines IL-7 and IL-15 were assessed to evaluate the impact of lymphodepleting chemotherapy. We found a significant increase in serum levels of IL-15 following preparative lymphodepleting chemotherapy but no increase in the level of IL-7 (Fig. 2a). These data are consistent with a previous study that found that the cyclophosphamide and fludarabine regimen increased serum IL-15 levels, but the addition of total body radiation was required to enhance serum IL-7 levels [10]. The observed increase in serum IL-15, however, indicated that lymphodepletion was effective and there was increased availability of this homeostatic cytokine for the infused TIL.

The serum level of IL-2 was also monitored. After TIL infusion, serum levels of IL-2 increased significantly a
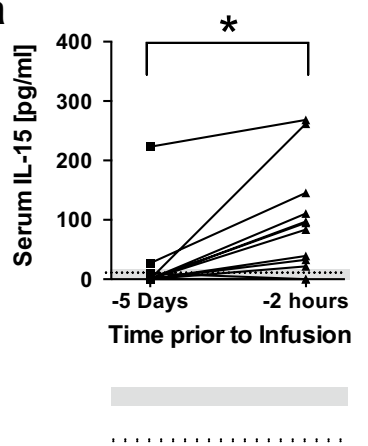

C

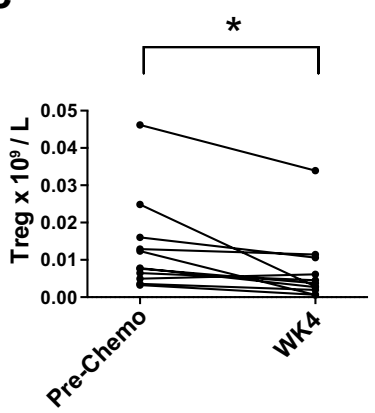

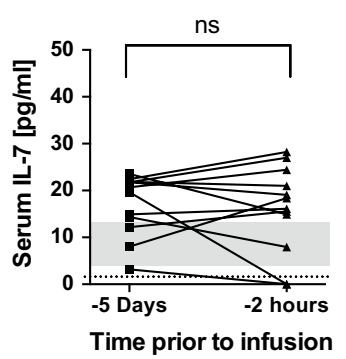

Healthy Donor

Limit of Detection b

్ㅗำ

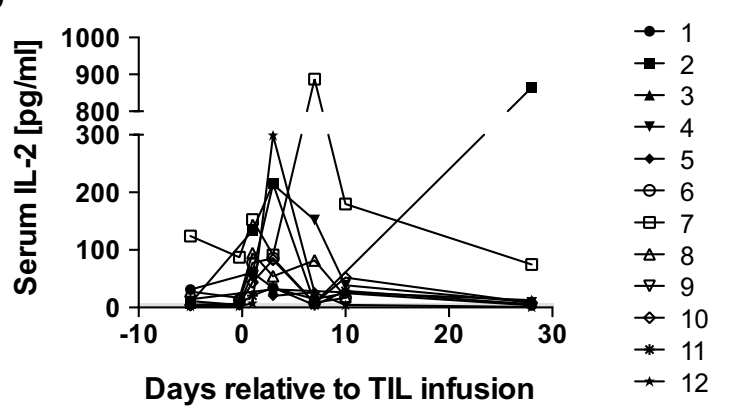

d

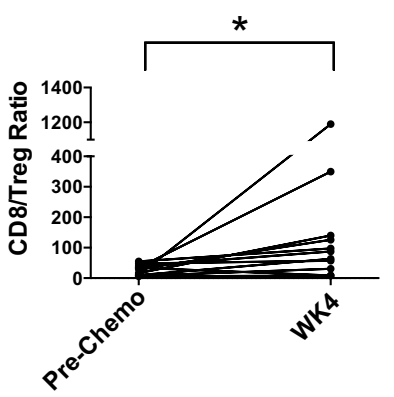

e

\section{WK65}

PD-1

Isotype

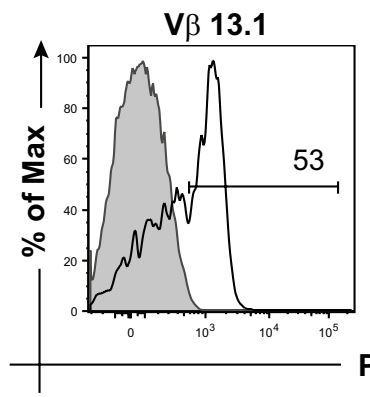

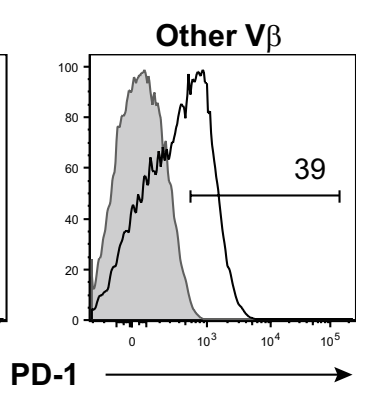

Fig. 2 Immune monitoring. a Serum samples were collected from each patient before lymphodepleting chemotherapy ( -5 days) and $2 \mathrm{~h}$ before TIL infusion and assayed by multiplex assay for IL-15 and IL-7. Shaded areas represent the range of serum cytokine concentration in healthy donors $(n=10)$ b Serum samples were collected from each patient at the indicated time points and assayed for IL-2 using a multiplex assay. c Number of regulatory $\mathrm{T}$ cells per liter of blood as well as the ratio of $\mathrm{CD}^{+} \mathrm{T}$ cells/Tregs before chemotherapy and
4 weeks after TIL infusion was quantified. Tregs were defined as $\mathrm{CD} 3{ }^{+} \mathrm{CD} 4{ }^{+} \mathrm{CD} 25^{\text {high }} \mathrm{CD} 127^{\text {low }} \mathrm{FoxP} 3^{+}$cells. d For Patient 1 , the percentage of $\mathrm{V} \beta 13.1^{+} \mathrm{T}$ cells in the $\mathrm{CD} 8^{+}$compartment in the TIL infusion product and in peripheral blood at the indicated time points was analyzed. e Peripheral blood of Patient 1 at 65 weeks after TIL infusion was analyzed for PD-1 expression, gated on $\mathrm{CD}^{+} \mathrm{V} \beta 13.1^{+} \mathrm{T}$ cells (left) or $\mathrm{CD}^{+} \mathrm{V} \beta 13.1^{-} \mathrm{T}$ cells (right) 
(Fig. 2b). This increased IL-2 was most evident during the first $72 \mathrm{~h}$ following TIL infusion, during which time the patients were also being treated with subcutaneous IL-2. These data suggest that the exogenous IL-2 was given at a sufficient dose to alter IL-2 levels in the circulation. Alternatively, it is also possible that the infused TIL themselves were producing IL-2 and were partially responsible for the increase observed. Regardless of source, an increase in serum IL-2 levels was clearly observed following TIL infusion on this treatment protocol. Although thought to be beneficial for the expansion of the transferred TIL, exogenous IL-2 has also been implicated in the preferential expansion of peripheral $\mathrm{CD} 4{ }^{+} \mathrm{FoxP}^{+}{ }^{+}$regulatory T cells (Tregs) [21]. Accordingly, we monitored Tregs in the peripheral blood and observed the inverse: a decrease in the absolute number of Tregs and an increase in the ratio of $\mathrm{CD}^{+}$cells to Tregs and (Fig. 2c). Thus, it appeared that all patients had increased serum levels of IL-2 without peripheral expansion of Tregs.

Next, we characterized the expanded TIL to determine whether any characteristics were associated with clinical response. The anti-tumor reactivity of TIL was assayed by two different approaches. First, we tested HLA class I-dependent interferon- $\gamma($ IFN- $\gamma)$ production as well as the class I-dependent cytolytic ability of the TIL cultures against autologous tumor (or for Patient 9, against HLA-A-matched tumor cells). We were able to test these in vitro functions in 10 of the 12 patients. However, we did not observe a discernable pattern to these results that was related to clinical response (Table 4). Amongst the responding patients, the TIL cultures from Patient 1 both produced IFN- $\gamma$ in response to autologous tumor, while those from Patient 8 did not. These assays were not performed for Patient 7 (PRu). In addition, many of the TIL cultures from the non-responding patients produced IFN- $\gamma$ and/or demonstrated cytolytic activity. Thus, in our cohort, these in vitro assays of antitumor activity were not predictive of response. Second, we determined the percentage of $\mathrm{CD}^{+} \mathrm{T}$ cells in the infusion product of each HLA-A*02:01-positive patient that recognized the tumor-associated antigen, Melanoma-associated antigen recognized by T cells (MART-1/Melan-A). Eight of the 12 patients were HLA-A*02:01-positive, and amongst those, the percentage of MART-1/Melan-A reactive cells in the infusion product was more than ninefold higher than in the baseline blood samples in six of the eight patients (Table 4). The two patients who had a confirmed PR did not show an enrichment of MART-1/Melan-A-reactive CD8 ${ }^{+} \mathrm{T}$ cells in the TIL infusion product. Thus, recognition of the HLA-A*02:01 MART-1/Melan-A epitope was not associated with clinical response. In the study reported by Radvanyi et al. [3], there was an association between the total number of $\mathrm{CD} 8^{+}$TIL infused and clinical response. We did not observe this association in our cohort, although interestingly, the three responding patients all had a dominance of $\mathrm{CD}^{+} \mathrm{T}$ cells in the infusion product (Table 2).

The presence of various T-cell receptor (TCR) beta chain variable region $(\mathrm{V} \beta)$ domains in the infusion products, as well as in peripheral blood samples taken before and after

Table 4 Tumor reactivity of TIL

\begin{tabular}{|c|c|c|c|c|c|c|c|}
\hline Patients & HLA-A & IFN- $\gamma^{\mathrm{a}}$ & Cytotoxicity $^{\mathrm{a}}$ & $\begin{array}{l}\% \text { MART- } 1 \text { base- } \\
\text { line PBMC }\end{array}$ & $\begin{array}{l}\% \text { MART- } 1 \text { infu- } \\
\text { sion product }^{b}\end{array}$ & $\begin{array}{l}\% \text { MART-1 Week } \\
1 \text { PBMC }^{\text {b }}\end{array}$ & $\begin{array}{l}\text { \%MART-1 } \\
\text { Week } 4 \\
\text { PBMC }^{\text {b }}\end{array}$ \\
\hline 1 & 0201/0201 & $2 / 2$ & $1 / 2$ & 0.03 & 0 & 0 & 0 \\
\hline 2 & 0101/0301 & $2 / 2$ & $2 / 2$ & N/A & N/A & N/A & N/A \\
\hline 3 & $0201 / 2403$ & $0 / 2$ & $2 / 2$ & 0.30 & 2.92 & 1.68 & 0.42 \\
\hline 4 & $0201 / 32$ & Not done & Not done & 0.17 & 5.23 & Not done & 1.26 \\
\hline 5 & $0201 / 2402$ & $2 / 2$ & $2 / 2$ & 0.04 & 58.5 & 56.7 & 0.04 \\
\hline 6 & $11 / 33$ & $0 / 2$ & $0 / 2$ & N/A & N/A & N/A & N/A \\
\hline 7 & 0101/0101 & Not done & Not done & N/A & N/A & N/A & N/A \\
\hline 8 & $0201 / 68$ & $0 / 2$ & $1 / 2$ & 0.32 & 0.35 & 0.18 & 0.52 \\
\hline 9 & 0201/0301 & $2 / 2$ & $2 / 2$ & 0.06 & 0.54 & 0.16 & 0.21 \\
\hline 10 & 0201/0301 & $1 / 2$ & $2 / 2$ & 0 & 0.11 & 0 & 0 \\
\hline 11 & $0201 / 23$ & $0 / 2^{c}$ & $0 / 2$ & 0.03 & 0.33 & 0 & 0 \\
\hline 12 & $0101 / 23$ & $0 / 2$ & $1 / 2$ & N/A & N/A & N/A & N/A \\
\hline
\end{tabular}

$T I L$ tumor-infiltrating lymphocyte, $P B M C$ peripheral blood mononuclear cell

${ }^{a}$ Of the TIL cultures used for REP: number of reactive TIL cultures/number of TIL cultures assayed

b\% MART-1 was defined as the percentage of CD8 + cells binding HLA-A*02:01/MART-1 multimers. Only patients expressing HLA-A*02:01 were evaluated for MART-1/Melan-A specificity

${ }^{\mathrm{c}}$ One TIL culture from Patient 11 exhibited IFN- $\gamma$ activity, but it was not reduced in the presence of anti-HLA Class I antibody 
TIL infusion, was analyzed using flow cytometry. To use $\mathrm{V} \beta$ repertoire as a surrogate indicator of engraftment of the TIL infusion product, we asked whether there was evidence of one or more dominant $\mathrm{V} \beta$ populations in the infusion product, and if so, whether the dominant population(s) could be found in blood after infusion. Eight patients had one or more dominant $\mathrm{V} \beta$ populations in the $\mathrm{CD} 8^{+}$compartment of their infusion product (Supplementary Table 1). Of these eight patients, two patients had the same $\mathrm{V} \beta$ populations at 1 and/or 4 weeks after infusion that were present in their infusion product. Four patients had the same $\mathrm{V} \beta$ populations at 1 week (but not at 4 weeks) after infusion that were present in their infusion product. In two patients, the $\mathrm{V} \beta$ population that was present in the infusion product was no longer dominant at 1 week or 4 weeks after infusion. The $\mathrm{CD} 4^{+}$compartments of the infusion products had far fewer dominant $\mathrm{V} \beta$ populations compared to the $\mathrm{CD} 8^{+}$compartments (Supplementary Table 2).

Collectively, the V $\beta$ flow cytometry data provide indirect evidence that the TIL product was capable of engrafting. Notably, one patient (Patient 1) had a dominant V $\beta 13.1$ population in the infusion product $\left(52 \%\right.$ of $\mathrm{CD} 8^{+} \mathrm{T}$ cells), and then also in blood at 1 week after infusion (76\%) which was still present at high levels at time points long after infusion (Fig. 2d and Supplementary Fig. 2). Interestingly, this patient had a dominant $\mathrm{V} \beta 16$ population that was detected 4 weeks after TIL infusion and remained at high levels past 6 months post-infusion, despite the fact that $\mathrm{V} \beta 16$ was not enriched in the TIL infusion product (Supplementary Fig. 2). At week 65, we analyzed PD-1 expression on the V $\beta 13.1$ population which was also dominant in the TIL infusion product. At this time point, over $20 \%$ of the peripheral CD $8^{+}$ $\mathrm{T}$ cells expressed a V $\beta 13.1 \mathrm{TCR}$. We found that a higher percentage of these $\mathrm{V} \beta 13.1^{+}$cells were $\mathrm{PD}-1^{+}$as compared to non-V $\beta 13.1$-expressing peripheral $\mathrm{CD}^{+}$cells (Fig. 2e). Expression of PD- 1 on $\mathrm{CD} 8^{+} \mathrm{T}$ cells in the peripheral circulation of melanoma patients has been linked to tumor specificity of these cells [22]. Together, these data provide evidence that supports the possibility that there were tumor-specific cells in the infusion product that persisted in a responding patient for more than a year after TIL therapy.

\section{Discussion}

Here, we report on the results of a phase II trial of a modified TIL treatment protocol for patients with advanced melanoma, substituting low-dose, subcutaneous IL-2 for the standard high-dose intravenous administration. When response was assessed per RECIST v1.1 criteria, there were two confirmed PRs and one unconfirmed PR observed amongst the 12 patients treated. This rate of response met the pre-specified criteria to consider the treatment effective. Despite meeting the statistical threshold for efficacy, a caveat to our results was that no patient achieved a CR and none of the PRs were durable. It is possible that the low response rate compared to other published clinical trials of TIL therapy for metastatic melanoma was due to the low-dose IL-2 regimen in our protocol; randomized trials would be needed to address this. It was also noted that 3 of the 12 patients treated in our study had mucosal or ocular melanoma. The response rate to TIL therapy and immunotherapy appears to be lower in these subsets [23-25]. None of these three patients responded in our study; therefore, the response rate of patients with cutaneous melanoma was $22 \%$ (2/9).

The observed toxicity attributable to the IL-2 was acceptable, with the majority of adverse events being grade 1 or grade 2 . These toxicities were managed in a non-ICU setting and no patients experienced broad cardiovascular collapse; this is in contrast to patients treated with high-dose IL-2 therapy. Toxicities generally could be reversed with supportive measures and/or by delaying or omitting subsequent IL-2 injections. The toxicity profile observed from the subcutaneous, low-dose IL-2 used in this trial is similar to the previous report of a TIL protocol that utilized a decrescendo intravenous dosing strategy [14]. Thus, low-dose IL-2 regimens may represent alternatives to high-dose IL-2 in future trials, especially when TIL therapy is combined with other immunotherapies such as immune checkpoint blockade.

Persistence of the transferred $\mathrm{T}$ cells has been strongly associated with response to TIL therapy $[2,14]$. Therefore, a lack of persistence of the infused TIL is another possible explanation for the lack of durable responses observed in our study. However, in addition to the suggestion of persistence of the transferred cells in Patient 1 (Fig. 2d), we also observed enrichment of MART-1/Melan-Aspecific $T$ cells in some of the non-responding patients, particularly Patients 4 and 9, a month after TIL transfer (Table 4). Recent data from Donia et al. have suggested that the persistence of the transferred cells alone may not be sufficient to provide benefit from TIL therapy and that tumor regression requires the accumulation of polyfunctional, PD- $1^{+} \mathrm{T}$ cells in the peripheral circulation after TIL infusion [26]. This observation is in accordance with our observation of high PD- 1 expression on the V $\beta 13.1^{+}$ $\mathrm{T}$ cells in Patient 1 (a responder) (Fig. 2e). It also supports an emerging paradigm that these peripheral PD- $1^{+} \mathrm{T}$ cells target tumor neo-antigens and that response to ACT correlates with the mutational burden of the tumor and the recognition of tumor neo-antigens, as opposed to shared tumor-associated antigens, by the transferred TIL [22, $27,28]$. Tumor neo-antigen load and mutational burden have also been correlated with response to anti-CTLA-4 therapy and overall survival with anti-PD-1 treatment in patients with melanoma [29-31]. Given that 11 of the 12 
patients treated in our trial had previously progressed on at least one line of immune checkpoint inhibitor therapy (Table 1), it is possible that our cohort included patients, whose tumors possessed relatively low neo-antigen loads. Studies are ongoing to further test this hypothesis by elucidating the antigen specificity of the transferred TIL as well as the mutational landscape of the patients' tumors.

If PD- $1^{+} \mathrm{CD} 8^{+} \mathrm{T}$ cells targeting tumor neo-antigens are indeed the major mediators of response to TIL therapy, this would also suggest that these cells are susceptible to regulation via the PD-1/PD-L1 axis. Interestingly, both the patients with confirmed PRs on this study, Patients 1 and 8 , subsequently went on to have durable responses to anti-PD-1 therapy after progression on TIL therapy. This was particularly notable for Patient 8 as his disease had progressed previously on anti-PD-1 therapy before TIL treatment, suggesting a potential synergy between the transferred TIL and the subsequent anti-PD-1 therapy. In addition, a dense infiltrate of PD $-1^{+} \mathrm{CD} 8^{+} \mathrm{T}$ cells was observed in a biopsy from a progressing cutaneous lesion post-TIL therapy in the patient with a PRu by RECIST (Patient 7) (Supplementary Fig. 3). It is tempting to speculate that treatment with anti-PD-1 could have re-invigorated these infiltrating cells and led to prolonged tumor regression.

Our experience with this small cohort of patients supports the rationale for future studies that combine TIL therapy with subsequent anti-PD-1/PD-L1 treatment. Currently, we are accruing patients who have progressed on previous anti-PD-1/PD-L1 therapy to a trial of ACT that incorporates both low dose, subcutaneous IL-2 and anti-PD-1 therapy after TIL infusion (NCT03158935). The goal of this trial is to improve the depth and durability of responses to our ACT regimen whilst still limiting the treatment-related toxicities of IL-2.

Acknowledgements We thank Dr. Nicole Liadis for coordination, and Dr. Azin Sayad for expert advice. We also thank the Cell Processing Lab of the Princess Margaret Cancer Centre Bone Marrow Transplant program. We are indebted to the blood donors and the patients who participated in this study, as well as their families.

Author Contributions Study conception and design: LTN, LK, AMJ, ES, SDL, LW, PSO, and MOB. Patient recruitment and management: SDS, LK, DG, LB, HM, DH, AMJ, MC, NF, AS, AH, AA, WL, AE, MR, DPG, DMC, KY, TW, MC, AP, BZ, SB-H, JC, and MOB. Data acquisition, management, analysis, and interpretation: LTN, SDS, VS, MXL, LB, MN, MF, CSL, JN, JYY, PHY, DG, VM, ARE, LW, SE, RE, NH, and MOB. Manuscript preparation: LTN, SDS, VS, MXL, $\mathrm{LB}, \mathrm{NH}$, and MOB.

Funding Pamela S. Ohashi holds a Tier 1 Canada Research Chair in Autoimmunity and Tumour Immunity and a Canadian Institutes of Health Research (CIHR) Foundation grant.
Data Availability The portions of the datasets generated or analyzed during the current study that are contained within confidential patient records are not publicly available, but the data that support the findings of this study that do not contain any identifiable information are available on reasonable request from the corresponding author.

\section{Com pliance with ethical standards}

Conflict of interest Anna Spreafico has served on Advisory Boards of: MERCK, Bristol-Myers Squibb, Novartis and Oncorus. Naoto Hirano reports receiving a research grant from and is a consultant for Takara Bio. Marcus O. Butler has served on Advisory Boards of: MERCK, Bristol-Myers Squibb, Novartis, Immunocore, Immunovaccine, EMD Serono and GlaxoSmithKline. Marcus O. Butler has received funding to support investigator initiated clinical trials from MERCK and Takara Bio. All other authors declare that they have no conflict of interest.

Ethical approval This study was registered on clinicatrials.gov (NCT01883323). All procedures performed in studies involving human participants were in accordance with the ethical standards of the institution (University Health Network Research Ethics Board approved protocol UHN REB\# 11-0683) and/or national research committees and with the 1964 Helsinki declaration and its later amendments or comparable ethical standards.

Informed consent Informed consent for protocol therapy, analysis of patient samples and publication was obtained from all individual participants included in the study.

OpenAccess This article is distributed under the terms of the Creative Commons Attribution 4.0 International License (http://creativeco mmons.org/licenses/by/4.0/), which permits unrestricted use, distribution, and reproduction in any medium, provided you give appropriate credit to the original author(s) and the source, provide a link to the Creative Commons license, and indicate if changes were made.

\section{References}

1. Rosenberg SA, Restifo NP (2015) Adoptive cell transfer as personalized immunotherapy for human cancer. Science 348(6230):6268. https://doi.org/10.1126/science.aaa4967

2. Rosenberg SA, Yang JC, Sherry RM, Kammula US, Hughes MS, Phan GQ, Citrin DE, Restifo NP, Robbins PF, Wunderlich JR, Morton KE, Laurencot CM, Steinberg SM, White DE, Dudley ME (2011) Durable complete responses in heavily pretreated patients with metastatic melanoma using T-cell transfer immunotherapy. Clin Cancer Res 17(13):4550-4557. https://doi.org/10.1158/10780432.CCR-11-0116

3. Radvanyi LG, Bernatchez C, Zhang M, Fox PS, Miller P, Chacon J, Wu R, Lizee G, Mahoney S, Alvarado G, Glass M, Johnson VE, McMannis JD, Shpall E, Prieto V, Papadopoulos N, Kim K, Homsi J, Bedikian A, Hwu WJ, Patel S, Ross MI, Lee JE, Gershenwald JE, Lucci A, Royal R, Cormier JN, Davies MA, Mansaray R, Fulbright OJ, Toth C, Ramachandran R, Wardell S, Gonzalez A, Hwu P (2012) Specific lymphocyte subsets predict response to adoptive cell therapy using expanded autologous tumor-infiltrating lymphocytes in metastatic melanoma patients. Clin Cancer Res 18(24):6758-6770. https://doi.org/10.1158/10780432.CCR-12-1177

4. Pilon-Thomas S, Kuhn L, Ellwanger S, Janssen W, Royster E, Marzban S, Kudchadkar R, Zager J, Gibney G, Sondak VK, Weber J, Mule JJ, Sarnaik AA (2012) Efficacy of adoptive cell transfer 
of tumor-infiltrating lymphocytes after lymphopenia induction for metastatic melanoma. J Immunother 35(8):615-620. https://doi. org/10.1097/CJI.0b013e31826e8f5f

5. Besser MJ, Shapira-Frommer R, Itzhaki O, Treves AJ, Zippel DB, Levy D, Kubi A, Shoshani N, Zikich D, Ohayon Y, Ohayon D, Shalmon B, Markel G, Yerushalmi R, Apter S, Ben-Nun A, Ben-Ami E, Shimoni A, Nagler A, Schachter J (2013) Adoptive transfer of tumor-infiltrating lymphocytes in patients with metastatic melanoma: intent-to-treat analysis and efficacy after failure to prior immunotherapies. Clin Cancer Res 19(17):4792-4800. https://doi.org/10.1158/1078-0432.CCR-13-0380

6. Goff SL, Dudley ME, Citrin DE, Somerville RP, Wunderlich JR, Danforth DN, Zlott DA, Yang JC, Sherry RM, Kammula US, Klebanoff CA, Hughes MS, Restifo NP, Langhan MM, Shelton TE, Lu L, Kwong ML, Ilyas S, Klemen ND, Payabyab EC, Morton KE, Toomey MA, Steinberg SM, White DE, Rosenberg SA (2016) Randomized, prospective evaluation comparing intensity of lymphodepletion before adoptive transfer of tumor-infiltrating lymphocytes for patients with metastatic melanoma. J Clin Oncol 34(20):2389-2397. https://doi.org/10.1200/JCO.2016.66.7220

7. Dudley ME, Wunderlich JR, Yang JC, Sherry RM, Topalian SL, Restifo NP, Royal RE, Kammula U, White DE, Mavroukakis SA, Rogers LJ, Gracia GJ, Jones SA, Mangiameli DP, Pelletier MM, Gea-Banacloche J, Robinson MR, Berman DM, Filie AC, Abati A, Rosenberg SA (2005) Adoptive cell transfer therapy following non-myeloablative but lymphodepleting chemotherapy for the treatment of patients with refractory metastatic melanoma. J Clin Oncol 23(10):2346-2357. https://doi.org/10.1200/ JCO.2005.00.240

8. Gattinoni L, Finkelstein SE, Klebanoff CA, Antony PA, Palmer DC, Spiess PJ, Hwang LN, Yu Z, Wrzesinski C, Heimann DM, Surh CD, Rosenberg SA, Restifo NP (2005) Removal of homeostatic cytokine sinks by lymphodepletion enhances the efficacy of adoptively transferred tumor-specific CD8+ T cells. J Exp Med 202(7):907-912. https://doi.org/10.1084/jem.20050732

9. Klebanoff CA, Khong HT, Antony PA, Palmer DC, Restifo NP (2005) Sinks, suppressors and antigen presenters: how lymphodepletion enhances $\mathrm{T}$ cell-mediated tumor immunotherapy. Trends Immunol 26(2):111-117. https://doi.org/10.1016/j.it.2004.12.003

10. Dudley ME, Yang JC, Sherry R, Hughes MS, Royal R, Kammula U, Robbins PF, Huang J, Citrin DE, Leitman SF, Wunderlich J, Restifo NP, Thomasian A, Downey SG, Smith FO, Klapper J, Morton K, Laurencot C, White DE, Rosenberg SA (2008) Adoptive cell therapy for patients with metastatic melanoma: evaluation of intensive myeloablative chemoradiation preparative regimens. J Clin Oncol 26(32):5233-5239. https://doi.org/10.1200/ JCO.2008.16.5449

11. Rosenberg SA (2014) Il-2: the first effective immunotherapy for human cancer. J Immunol 192(12):5451-5458. https://doi. org/10.4049/jimmunol.1490019

12. Ullenhag GJ, Sadeghi AM, Carlsson B, Ahlstrom H, Mosavi F, Wagenius G, Totterman TH (2012) Adoptive T-Cell therapy for malignant melanoma patients with tils obtained by ultrasoundguided needle biopsy. Cancer Immunol Immunother 61(5):725732. https://doi.org/10.1007/s00262-011-1182-4

13. Ellebaek E, Iversen TZ, Junker N, Donia M, Engell-Noerregaard L, Met O, Holmich LR, Andersen RS, Hadrup SR, Andersen MH, thor Straten P, Svane IM (2012) Adoptive cell therapy with autologous tumor infiltrating lymphocytes and low-dose interleukin-2 in metastatic melanoma patients. J Transl Med 10:169. https://doi. org/10.1186/1479-5876-10-169

14. Andersen R, Donia M, Ellebaek E, Borch TH, Kongsted P, Iversen TZ, Holmich LR, Hendel HW, Met O, Andersen MH, Thor Straten P, Svane IM (2016) Long-lasting complete responses in patients with metastatic melanoma after adoptive cell therapy with tumorinfiltrating lymphocytes and an attenuated $\mathrm{I} 22$ regimen. Clin
Cancer Res 22(15):3734-3745. https://doi.org/10.1158/10780432.CCR-15-1879

15. Balch CM, Gershenwald JE, Soong SJ, Thompson JF, Atkins MB, Byrd DR, Buzaid AC, Cochran AJ, Coit DG, Ding S, Eggermont AM, Flaherty KT, Gimotty PA, Kirkwood JM, McMasters KM, Mihm MC Jr, Morton DL, Ross MI, Sober AJ, Sondak VK (2009) Final version of 2009 AJCC melanoma staging and classification. J Clin Oncol 27(36):6199-6206. https://doi.org/10.1200/ JCO.2009.23.4799

16. Eisenhauer EA, Therasse P, Bogaerts J, Schwartz LH, Sargent D, Ford R, Dancey J, Arbuck S, Gwyther S, Mooney M, Rubinstein L, Shankar L, Dodd L, Kaplan R, Lacombe D, Verweij J (2009) New response evaluation criteria in solid tumours: revised recist guideline (Version 1.1). Eur J Cancer 45(2):228-247. https://doi. org/10.1016/j.ejca.2008.10.026

17. Wolchok JD, Hoos A, O'Day S, Weber JS, Hamid O, Lebbe C, Maio M, Binder M, Bohnsack O, Nichol G, Humphrey R, Hodi FS (2009) Guidelines for the evaluation of immune therapy activity in solid tumors: immune-related response criteria. Clin Cancer Res 15(23):7412-7420. https://doi.org/10.1158/10780432.CCR-09-1624

18. Yang JC, Sherry RM, Steinberg SM, Topalian SL, Schwartzentruber DJ, Hwu P, Seipp CA, Rogers-Freezer L, Morton KE, White DE, Liewehr DJ, Merino MJ, Rosenberg SA (2003) Randomized study of high-dose and low-dose interleukin-2 in patients with metastatic renal cancer. J Clin Oncol 21(16):31273132. https://doi.org/10.1200/jco.2003.02.122

19. Tran KQ, Zhou J, Durflinger KH, Langhan MM, Shelton TE, Wunderlich JR, Robbins PF, Rosenberg SA, Dudley ME (2008) Minimally cultured tumor-infiltrating lymphocytes display optimal characteristics for adoptive cell therapy. J Immunother 31(8):742-751. https://doi.org/10.1097/CJI.0b013e31818403d 5

20. Nguyen LT, Yen PH, Nie J, Liadis N, Ghazarian D, Al-Habeeb A, Easson A, Leong W, Lipa J, McCready D, Reedijk M, Hogg D, Joshua AM, Quirt I, Messner H, Shaw P, Crump M, Sharon E, Ohashi PS (2010) Expansion and characterization of human melanoma tumor-infiltrating lymphocytes (TILs). PLoS One 5(11):e13940. https://doi.org/10.1371/journal.pone.0013940

21. Yao X, Ahmadzadeh M, Lu YC, Liewehr DJ, Dudley ME, Liu F, Schrump DS, Steinberg SM, Rosenberg SA, Robbins PF (2012) Levels of peripheral CD4(+)Foxp3(+) regulatory T cells are negatively associated with clinical response to adoptive immunotherapy of human cancer. Blood 119(24):5688-5696. https://doi. org/10.1182/blood-2011-10-386482

22. Gros A, Parkhurst MR, Tran E, Pasetto A, Robbins PF, Ilyas S, Prickett TD, Gartner JJ, Crystal JS, Roberts IM, TrebskaMcGowan K, Wunderlich JR, Yang JC, Rosenberg SA (2016) Prospective identification of neoantigen-specific lymphocytes in the peripheral blood of melanoma patients. Nat Med 22(4):433-438. https://doi.org/10.1038/nm.4051

23. Algazi AP, Tsai KK, Shoushtari AN, Munhoz RR, Eroglu Z, Piulats JM, Ott PA, Johnson DB, Hwang J, Daud AI, Sosman JA, Carvajal RD, Chmielowski B, Postow MA, Weber JS, Sullivan RJ (2016) Clinical outcomes in metastatic uveal melanoma treated with PD-1 and PD-L1 antibodies. Cancer 122(21):3344-3353. https://doi.org/10.1002/cncr.30258

24. D’Angelo SP, Larkin J, Sosman JA, Lebbe C, Brady B, Neyns B, Schmidt H, Hassel JC, Hodi FS, Lorigan P, Savage KJ, Miller WH Jr, Mohr P, Marquez-Rodas I, Charles J, Kaatz M, Sznol M, Weber JS, Shoushtari AN, Ruisi M, Jiang J, Wolchok JD (2017) Efficacy and safety of nivolumab alone or in combination with ipilimumab in patients with mucosal melanoma: a pooled analysis. J Clin Oncol 35(2):226-235. https://doi.org/10.1200/jco.2016.67.9258

25. Hamid O, Robert C, Ribas A, Hodi FS, Walpole E, Daud A, Arance AS, Brown E, Hoeller C, Mortier L, Schachter J, Long J, 
Ebbinghaus S, Ibrahim N, Butler M (2018) Antitumour activity of pembrolizumab in advanced mucosal melanoma: a post-hoc analysis of keynote-001, 002, 006. Br J Cancer. https://doi.org/10.1038/ s41416-018-0207-6

26. Donia M, Kjeldsen JW, Andersen R, Westergaard MCW, Bianchi V, Legut M, Attaf M, Szomolay B, Ott S, Dolton G, Lyngaa R, Hadrup SR, Sewell AK, Svane IM (2017) PD-1(+) polyfunctional $\mathrm{T}$ cells dominate the periphery after tumor-infiltrating lymphocyte therapy for cancer. Clin Cancer Res 23(19):5779-5788. https:// doi.org/10.1158/1078-0432.CCR-16-1692

27. Robbins PF, Lu YC, El-Gamil M, Li YF, Gross C, Gartner J, Lin JC, Teer JK, Cliften P, Tycksen E, Samuels Y, Rosenberg SA (2013) Mining exomic sequencing data to identify mutated antigens recognized by adoptively transferred tumor-reactive $\mathrm{T}$ cells. Nat Med 19(6):747-752. https://doi.org/10.1038/nm.3161

28. Lauss M, Donia M, Harbst K, Andersen R, Mitra S, Rosengren F, Salim M, Vallon-Christersson J, Torngren T, Kvist A, Ringner M, Svane IM, Jonsson G (2017) Mutational and putative neoantigen load predict clinical benefit of adoptive $\mathrm{T}$ cell therapy in melanoma. Nat Commun 8(1):1738. https://doi.org/10.1038/s4146 7-017-01460-0
29. Snyder A, Makarov V, Merghoub T, Yuan J, Zaretsky JM, Desrichard A, Walsh LA, Postow MA, Wong P, Ho TS, Hollmann TJ, Bruggeman C, Kannan K, Li Y, Elipenahli C, Liu C, Harbison CT, Wang L, Ribas A, Wolchok JD, Chan TA (2014) Genetic basis for clinical response to CTLA-4 blockade in melanoma. N Engl J Med 371(23):2189-2199. https://doi.org/10.1056/NEJMoa1406 498

30. Van Allen EM, Miao D, Schilling B, Shukla SA, Blank C, Zimmer L, Sucker A, Hillen U, Foppen MHG, Goldinger SM, Utikal J, Hassel JC, Weide B, Kaehler KC, Loquai C, Mohr P, Gutzmer R, Dummer R, Gabriel S, Wu CJ, Schadendorf D, Garraway LA (2015) Genomic correlates of response to CTLA-4 blockade in metastatic melanoma. Science 350(6257):207-211. https://doi. org/10.1126/science.aad0095

31. Hugo W, Zaretsky JM, Sun L, Song C, Moreno BH, Hu-Lieskovan S, Berent-Maoz B, Pang J, Chmielowski B, Cherry G, Seja E, Lomeli S, Kong X, Kelley MC, Sosman JA, Johnson DB, Ribas A, Lo RS (2016) Genomic and transcriptomic features of response to Anti-PD-1 therapy in metastatic melanoma. Cell 165(1):35-44. https://doi.org/10.1016/j.cell.2016.02.065

\section{Affiliations}

Linh T. Nguyen ${ }^{1}$ D . Samuel D. Saibil ${ }^{2} \cdot$ Valentin Sotov $^{1} \cdot$ Michael X. Le ${ }^{1} \cdot$ Leila Khoja $^{2} \cdot$ Danny Ghazarian $^{3}$. Luisa Bonilla ${ }^{2} \cdot$ Habeeb Majeed $^{2} \cdot$ David Hogg $^{2} \cdot$ Anthony M. Joshua $^{2,4} \cdot$ Michael Crump $^{2} \cdot$ Norman Franke $^{2}$. Anna Spreafico ${ }^{2} \cdot$ Aaron Hansen $^{2} \cdot$ Ayman Al-Habeeb $^{3} \cdot$ Wey Leong $^{5} \cdot$ Alexandra Easson $^{5} \cdot$ Michael Reedijk $^{5}$. David P. Goldstein ${ }^{5,6} \cdot$ David McCready $^{5} \cdot$ Kazuhiro Yasufuku $^{5} \cdot$ Thomas Waddell $^{5} \cdot$ Marcelo Cypel $^{5} \cdot$ Andrew Pierre $^{5}$. Bianzheng Zhang ${ }^{2}$. Sarah Boross-Harmer ${ }^{1}$. Jane Cipollone ${ }^{1} \cdot$ Megan Nelles $^{1}$ - Elizabeth Scheid ${ }^{1} \cdot$ Michael Fyrsta $^{1}$. Charlotte S. Lo ${ }^{1}$. Jessica Nie ${ }^{1}$. Jennifer Y. Yam ${ }^{1}$. Pei Hua Yen ${ }^{1}$. Diana Gray ${ }^{1}$. Vinicius Motta ${ }^{1}$ Alisha R. Elford ${ }^{1}$. Stephanie DeLuca ${ }^{7}$ Lisa Wang ${ }^{8} \cdot$ Stephanie Effendi $^{8} \cdot$ Ragitha Ellenchery $^{8} \cdot$ Naoto Hirano $^{1,9} \cdot$ Pamela S. Ohashi $^{1,9}$. Marcus O. Butler ${ }^{1,2,9,10}$

1 Tumor Immunotherapy Program, Princess Margaret Cancer Centre, Toronto, Canada

2 Department of Medical Oncology and Hematology, Princess Margaret Cancer Centre, Toronto, Canada

3 Department of Laboratory Medicine, University Health Network, Toronto, Canada

4 Kinghorn Cancer Centre, St. Vincent's Hospital, Sydney, Australia

5 Department of Surgical Oncology, Princess Margaret Cancer Centre, Toronto, Canada

6 Department of Otolaryngology, Head and Neck Surgery, Princess Margaret Cancer Centre, Toronto, Canada
7 Department of Pharmacy, Princess Margaret Cancer Centre, Toronto, Canada

8 Drug Development Program, Princess Margaret Cancer Centre, Toronto, Canada

9 Department of Immunology, University of Toronto, Toronto, Canada

10 University Health Network, Princess Margaret Cancer Centre, 9-622, 610 University Avenue, Toronto, ON M5G 2M9, Canada 